\title{
The development and on-orbit calibration status of GOSAT-2 TANSO-CAI-2 instrument
}

Takeru Uno, Toshiharu Oishi, Yasutaka Fujii, Masao Imaki, Aki Sato, et al.

Takeru Uno, Toshiharu Oishi, Yasutaka Fujii, Masao Imaki, Aki Sato, Fumie Kataoka, Hiroko Imai, Makiko Hashimoto, Kei Shiomi, Masakatsu Nakajima, "The development and on-orbit calibration status of GOSAT-2 TANSO-CAI-2 instrument," Proc. SPIE 11852, International Conference on Space Optics ICSO 2020, 1185257 (11 June 2021); doi: 10.1117/12.2599936

SPIE Event: International Conference on Space Optics - ICSO 2021, 2021, Online Only 


\section{International Conference on Space Optics-ICSO 2020}

Virtual Conference

30 March-2 April 2021

Edited by Bruno Cugny, Zoran Sodnik, and Nikos Karafolas
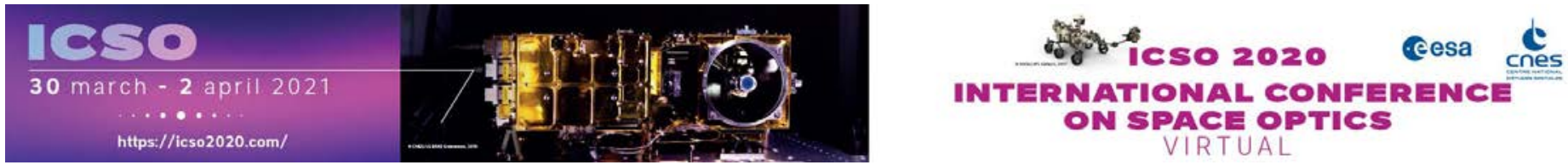

\section{The development and on-orbit calibration status of GOSAT-2 TANSO-CAI-2 instrument}

\section{Cesa isoporecestings denes}




\title{
The development and on-orbit calibration status of GOSAT-2 TANSO-CAI-2 instrument
}

\author{
Takeru Uno*a, Toshiharu Oishi ${ }^{\mathrm{a}}$, Yasutaka Fujii ${ }^{\mathrm{a}}$, Masao Imaki ${ }^{\mathrm{a}}$, Aki Sato ${ }^{\mathrm{b}}$, Fumie Kataoka ${ }^{\mathrm{b}}$, \\ Hiroko Imai ${ }^{\mathrm{c}}$, Makiko Hashimoto ${ }^{\mathrm{c}}$, Kei Shiomi ${ }^{\mathrm{c}}$, Masakatsu Nakajima ${ }^{\mathrm{c}}$ \\ ${ }^{a}$ Mitsubishi Electric Corporation, 325 Kamimachiya, Kamakura, Kanagawa, 247-8520 Japan; \\ ${ }^{b}$ Remote Sensing Technology Center of Japan, 2-1-1 Sengen, Tsukuba, Ibaraki, 305-8505 Japan; \\ ${ }^{\mathrm{c} J a p a n}$ Aerospace Exploration Agency, 2-1-1 Sengen, Tsukuba, Ibaraki, 305-8505 Japan.
}

\begin{abstract}
TANSO-CAI-2 onboard GOSAT-2 satellite is a multi-spectral imager for the observation of cloud and aerosol. The CAI2 has 10 observation bands with 7 different wavelengths, which cover 340-1630 nm. The wavelength width of each band is less than $13 \mathrm{~nm}$ in the UV to NIR band and less than $73 \mathrm{~nm}$ in the SWIR band. From Feb. 2019, the evaluation of the initial calibration phase was carried out to calibrate the geometric and radiometric characteristics. Following the initial calibration phase, the evaluation of radiometric performance has been continuously conducted. The evaluation of dark level and dark noise has shown almost stable results, although there is some variation. The radiometric response evaluation using the lunar calibration data and the ROLO model showed a degradation in the UV Bands, especially in Band 1 (340 $\mathrm{nm}$ ), which showed a degradation of about $8 \%$ in the two years relative to Band 4 ( $865 \mathrm{~nm}$ ). No obvious response degradation was observed for the other bands. Those results suggest that the radiometric performance of the CAI-2 is almost maintained for two years since the initial calibration phase.
\end{abstract}

Keywords: GOSAT-2, TANSO-CAI-2, Space Optics, Calibration, Radiometric calibration, Lunar calibration

\section{INTRODUCTION}

The Greenhouse gases Observing SATellite-2 (GOSAT-2) is a satellite to monitoring the greenhouse gases such as carbondioxide $\left(\mathrm{CO}_{2}\right)$ and methane $\left(\mathrm{CH}_{4}\right)^{1}$. The GOSAT-2 was launched successfully on October 29th, 2018. The GOSAT-2 has two mission instruments of the Thermal And Near infrared Sensor for carbon Observation - Fourier Transform Spectrometer-2 (TANSO-FTS-2; FTS-2) and the Thermal And Near infrared Sensor for carbon Observation - Cloud and Aerosol Imager-2 (TANSO-CAI-2; CAI-2). An overview image of the GOSAT-2 satellite and the two mission instruments is illustrated in Figure 1.

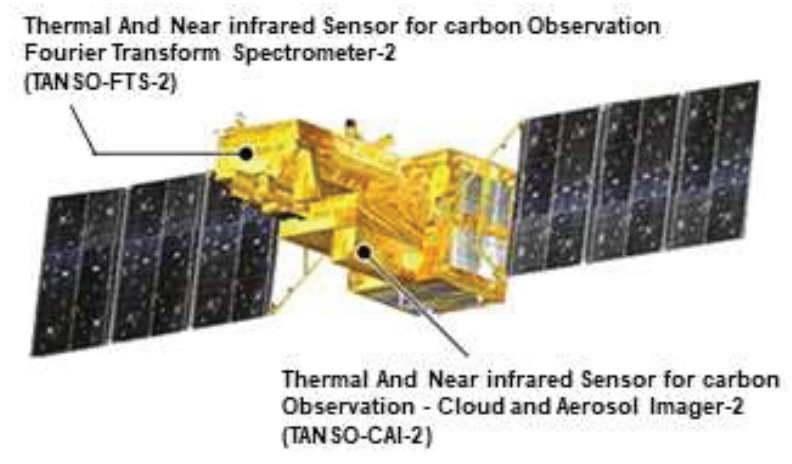

Figure 1. Overview image of the GOSAT-2 Satellite and two mission instruments of the FTS-2 and the CAI-2.

*Uno.Takeru@cw.MitsubishiElectric.co.jp 
The FTS-2 is a spectrometer in the short-wavelength infrared and thermal infrared regions, which are related to solar scattered light reflected from the earth's surface and atmospheric radiation, respectively. The CAI-2 is a multi-spectral imager with two purposes. One is to detect clouds, which are a cause of inaccuracy in the derivation of greenhouse gases using the FTS-2. The other is to derive the optical thickness of the aerosol, the content of particulate matter and black carbon.

The major specification of the FTS-2 is shown in Table 1. The FTS-2 is a 5-bands Fourier transform spectrometer sensing which can detect from short wavelength infrared (SWIR) range to thermal infrared (TIR) range ${ }^{2,3}$. It observes the density of $\mathrm{CO}_{2}, \mathrm{CH}_{4}, \mathrm{H}_{2} \mathrm{O}, \mathrm{O}_{2}, \mathrm{O}_{3}$ and $\mathrm{CO}$.

Table 1. Major specification of the FTS-2. All values in the table below indicate nominal values.

\begin{tabular}{|c|c|c|c|c|c|}
\hline Item & Band1 & Band2 & Band3 & Band4 & Band5 \\
\hline Polarization & $\mathrm{P}$ and $\mathrm{S}$ & $\mathrm{P}$ and $\mathrm{S}$ & $\mathrm{P}$ and $\mathrm{S}$ & No & No \\
\hline $\begin{array}{l}\text { Wavelength Range } \\
\left(\mathrm{cm}^{-1}\right)\end{array}$ & $12950-13250$ & $5900-6400$ & $4200-5200$ & $1188-1800$ & $700-1188$ \\
\hline $\begin{array}{c}\text { Out-of-band } \\
\text { Characteristics }\left(\mathrm{cm}^{-1}\right)\end{array}$ & $\begin{array}{l}<12750 \\
>13450 \\
\end{array}$ & $\begin{array}{l}<5100 \\
>6800 \\
\end{array}$ & $\begin{array}{l}<4100 \\
>5500 \\
\end{array}$ & $\begin{array}{l}<1000 \\
>3800 \\
\end{array}$ & $\begin{array}{c}<600 \\
>1300\end{array}$ \\
\hline $\begin{array}{l}\text { Spectral Resolution } \\
\left(\mathrm{cm}^{-1}\right)\end{array}$ & \multicolumn{5}{|c|}{0.2} \\
\hline $\begin{array}{l}\text { FWHM of Instrument } \\
\text { function }\left(\mathrm{cm}^{-1}\right)\end{array}$ & 0.4 & \multicolumn{4}{|c|}{0.27} \\
\hline $\begin{array}{l}\text { Aperture Diameter } \\
(\mathrm{mm})\end{array}$ & \multicolumn{5}{|c|}{0.77 (Effective Aperture Diameter) } \\
\hline FOV (mrad) & \multicolumn{5}{|c|}{15.8 (Angular Field of View) } \\
\hline
\end{tabular}

The major specification of the CAI-2 is shown in Table 2 and 3. The CAI-2 is a 7-wavelength/10-bands electronical scanning imager for ultraviolet, visible and near infrared. The wavelength width of each band is less than $13 \mathrm{~nm}$ for VIS to NIR band and less than $73 \mathrm{~nm}$ for SWIR band. The total wavelength range is $340-1630 \mathrm{~nm}$. The CAI-2 has 5 optical systems ("Telescopes") equipped with bands in the forward and backward directions. Bands 1 to 5 observe forward direction of $+20^{\circ}$ from nadir in flight direction (along-track direction: AT direction). Bands 6 to 10 observe backward direction of $-20^{\circ}$ from nadir in AT direction. With a wide field of view of more than $900 \mathrm{~km}$ in the direction perpendicular to the flight direction (cross-track direction: CT direction), it is possible to obtain the spatial distribution of clouds and aerosols. The optical axis of the CAI-2 is fixed on the satellite structure. The CAI-2 performs daytime global observations in 6 days. A schematic view of the observation of the CAI-2 is shown in Figure 2.

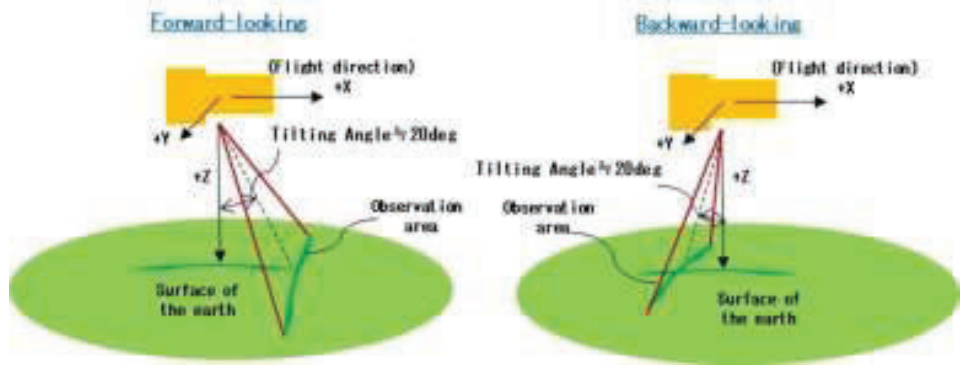

Figure 2. A schematic view of the observation of the CAI-2. The CAI-2 has two groups of observation bands, one is a forward-looking and the other is a backward-looking. 
Table 2. Major Specifications of the CAI-2 (Forward bands). The values shown in italics (center wavelength, wavelength range and swath) are measured values based on test results. Other values in the table below indicate nominal values. Note that the effective pixels of Band5 exclude the masked pixels.

\begin{tabular}{|c|c|c|c|c|c|}
\hline Item & Band 1 & Band 2 & Band 3 & Band 4 & Band 5 \\
\hline Optical Telescope & Telescope 1 & Telescope 2 & Telescope 3 & Telescope 4 & Telescope 5 \\
\hline Pointing Direction & \multicolumn{5}{|c|}{ Forward-looking $\left(+20^{\circ}\right.$ in AT direction) } \\
\hline Center Wavelength $(\mu \mathrm{m})$ & 0.339 & 0.441 & 0.672 & 0.865 & 1.630 \\
\hline Wavelength Width $(\mu \mathrm{m})$ & 0.013 & 0.012 & 0.013 & 0.011 & 0.073 \\
\hline $\begin{array}{l}\text { Resolution }(\mathrm{km}) \\
\text { (IFOV at the center of the field } \\
\text { of the view) }\end{array}$ & \multicolumn{4}{|c|}{0.46} & 0.92 \\
\hline $\begin{array}{c}\text { Swath }(\mathrm{km}) \\
\text { (FOV in CT direction) }\end{array}$ & \multicolumn{5}{|c|}{ Over $903\left( \pm 33.8^{\circ}\right)$} \\
\hline Type of Detector & \multicolumn{4}{|c|}{ Si CCD } & InGaAs \\
\hline Effective Pixels & \multicolumn{4}{|c|}{2048} & 958 \\
\hline Pre-Scan Pixels & \multicolumn{4}{|c|}{8} & - \\
\hline Pixel Pitch $(\mu \mathrm{m})$ & \multicolumn{4}{|c|}{14} & 25 \\
\hline
\end{tabular}

Table 3. Major Specifications of the CAI-2 (Backward bands). The values shown in italics (center wavelength, wavelength range and swath) are measured values based on test results. Other values in the table below indicate nominal values. Note that the effective pixels of Band10 exclude the masked pixels.

\begin{tabular}{|c|c|c|c|c|c|}
\hline Item & Band 6 & Band 7 & Band 8 & Band 9 & Band 10 \\
\hline Optical Telescope & Telescope 1 & Telescope 2 & Telescope 3 & Telescope 4 & Telescope 5 \\
\hline Pointing Direction & \multicolumn{5}{|c|}{ Backward-looking $\left(-20^{\circ}\right.$ in AT direction) } \\
\hline Center Wavelength $(\mu \mathrm{m})$ & 0.377 & 0.546 & 0.672 & 0.865 & 1.630 \\
\hline Wavelength Width $(\mu \mathrm{m})$ & 0.012 & 0.013 & 0.013 & 0.011 & 0.073 \\
\hline $\begin{array}{c}\text { Resolution }(\mathrm{km}) \\
\text { (IFOV at the center of the field } \\
\text { of the view) }\end{array}$ & \multicolumn{4}{|c|}{0.46} & 0.92 \\
\hline $\begin{array}{c}\text { Swath }(\mathrm{km}) \\
\text { (FOV in CT direction) }\end{array}$ & \multicolumn{5}{|c|}{ Over $903\left( \pm 33.8^{\circ}\right)$} \\
\hline Type of Detector & \multicolumn{4}{|c|}{ Si CCD } & InGaAs \\
\hline Effective Pixels & \multicolumn{4}{|c|}{2048} & 958 \\
\hline Pre-Scan Pixels & \multicolumn{4}{|c|}{8} & - \\
\hline Pixel Pitch $(\mu \mathrm{m})$ & \multicolumn{4}{|c|}{14} & 25 \\
\hline
\end{tabular}

\section{COMMISSIONING PHASE AND INITIAL CALIBRATION PHASE}

In three months after launch on October 29th, 2018, the initial checkout of the GOSAT-2 satellite bus system, the FTS-2 and the CAI-2 was conducted. Based on the results of the initial checkout evaluation, it was confirmed that all functions and performance of the CAI-2 satisfied the requirements ${ }^{4}$. Following the initial checkout phase, the initial calibration phase started in February 2019. In the initial calibration phase, the calibration data of geometric and radiometric characteristics were obtained, and the calibration parameters were adjusted.

\subsection{Geometric calibration}

To evaluate the geometric performance of the CAI-2, the observation of ground control point (GCP) and cross-correlation methods were used. At first, the absolute direction vector of each pixel in the reference bands (Band 4 and Band 9) derived using GCP was calculated. After that, the difference of the direction vector for the other bands with respect to the reference band was calculated using cross-correlation between images. The direction vector data ware calibrated using these observation data. 


\subsection{Radiometric performance}

In the radiometric calibration, the lunar calibration is performed using the Moon as the reference light source to evaluate the radiometric performance of the CAI-2. The schematic diagram of the lunar calibration is shown in Figure 3.
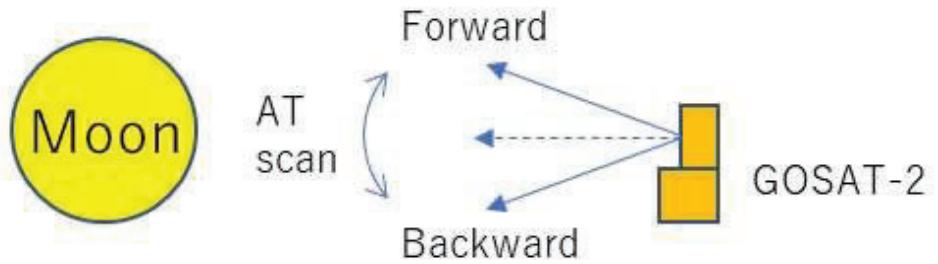

Figure 3. Schematic diagram of the CAI-2 lunar calibration. The satellite body is oriented to the Moon, and the Moon is scanned by controlling the satellite attitude in the AT direction.

As shown in Figure 3, the Moon image is obtained by scanning the field of view by controlling the satellite attitude in the Along Track (AT) direction. An example of the observed Moon image is shown in Figure 4. Due to the slow scanning, the Moon image is vertically elongated.

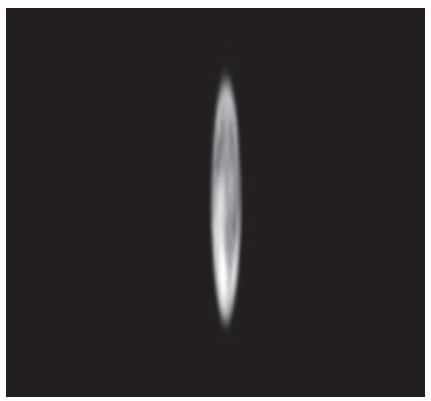

Figure 4. An example of the observed Moon image of Band 4 taken on December 2018.

The ROLO model ${ }^{5}$ was used as the lunar reflectance model, and the full disk integrated spectral irradiance of the Moon measured by the CAI-2 was compared with the model using the following procedure ${ }^{6}$ :

(1) In order to reduce the influence of the uncertainty of the Moon reflectance model, the lunar calibration is conducted by fixing the timing of the lunar phase angle to be around +7 degrees just after full moon.

(2) Obtained the full disk integrated spectral irradiance $H$ of the Moon from the image data of the Moon after radiance conversion. $H$ is obtained by multiplying the spectral radiance of the Moon at each pixel $\left(\mathrm{Wm}^{-2} \mathrm{sr}^{-1} \mu \mathrm{m}^{-1}\right)$ by the solid angle per pixel and the oversampling factor (about 0.125 in the case of the CAI-2 lunar calibration).

(3) The disk equivalent reflectance is derived from the ROLO model using geometric information such as the phase angles of the satellite, the Moon, and the Sun at the time of observation.

(4) Obtain a model of the full disk integrated spectral irradiance $H^{\prime}$ of the Moon using the disk equivalent reflectance, the spectral irradiance of the Sun, the distance between the Moon, the Sun, and the satellite.

(5) Compare with $H$ and $H^{\prime}$. 
The wavelength of the disk equivalent reflectance of the Moon obtained by the ROLO model is not identical with the wavelength of the CAI-2. The wavelengths of the ROLO model are discrete and only available at wavelengths from 347 $\mathrm{nm}$ to $2390 \mathrm{~nm}$. Therefore, the obtained reflectance data of the ROLO model was fitted with a logarithmic curve, extrapolated to $340 \mathrm{~nm}$, and replaced with the data corresponding to the observed wavelength of the CAI-2. The fitted reflectance data are shown in Figure 5. The approximation curve is slightly larger than that of the ROLO model in the range of 1000-1300 nm, but it is well approximated in the shorter wavelength range than $1000 \mathrm{~nm}$ and around $1600 \mathrm{~nm}$.
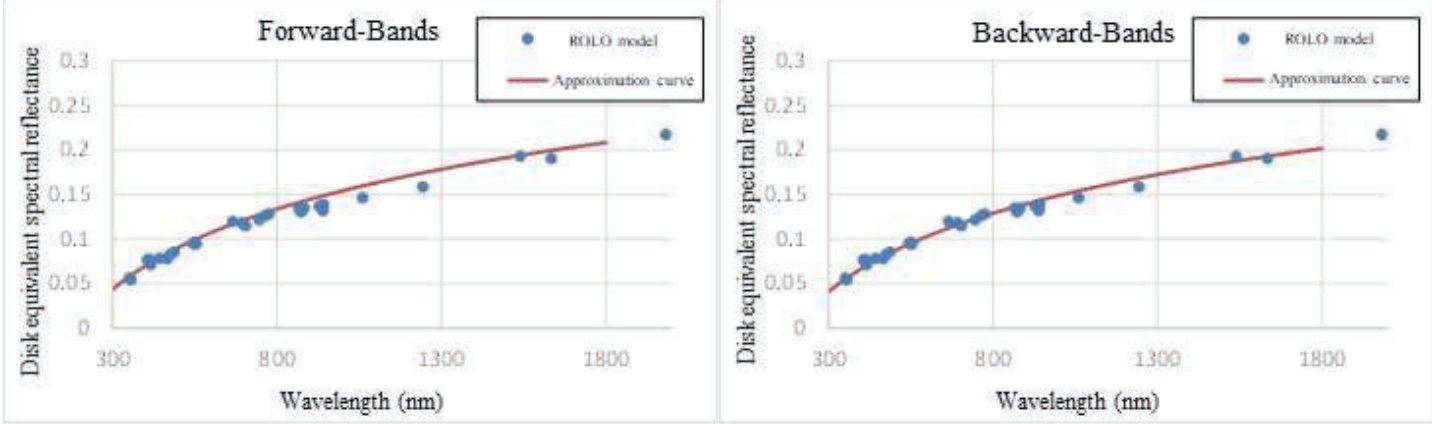

Figure 5. An example of the disk equivalent spectral reflectance of the Moon produced by the ROLO model and the approximation curve. (a) Forward-looking observation; (b) Backward-looking observation. The dots are reflectance of the ROLO model. The red lines are the approximation curve.

The irradiance model of the Moon $H^{\prime}$ was calculated from the disk equivalent reflectance and solar spectra. As the solar spectra, the solar spectral model of Wehrli (1986) ${ }^{7}$, which was used in the ROLO model, was used to avoid the influence of the uncertainty of the solar spectral irradiance model. The comparison between the observed lunar irradiance $H$ and the calculated irradiance $H$ ' based on the ROLO model is shown in Table 4. In Table 4, some bands show a deviation of up to $10 \%$. Such deviation could be caused by errors in the ground-based radiometric calibration, uncertainties in the satellite's orbital position and attitude, and uncertainties in the lunar reflectance model itself. According to previous study, the absolute error of the lunar reflectance model might be up to $5-10 \%{ }^{8}$.

Table 4. The comparison between the observed lunar irradiance and the calculated irradiance based on the ROLO model at the beginning of initial checkout phase (December 2018).

\begin{tabular}{|c|c|c|c|}
\hline Band & $\begin{array}{c}\text { Observed lunar } \\
\text { irradiance } \\
H\left(\mathrm{Wm}^{-2} \mu \mathrm{m}^{-1}\right)\end{array}$ & $\begin{array}{c}\text { Calculated lunar } \\
\text { irradiance model } \\
H^{\prime}\left(\mathrm{Wm}^{-2} \mu \mathrm{m}^{-1}\right)\end{array}$ & $\begin{array}{c}\text { Deviation } \\
\left(H-H^{\prime}\right) / H^{\prime}(\%)\end{array}$ \\
\hline Band1 & 0.00144 & 0.00133 & 8.8 \\
\hline Band2 & 0.00405 & 0.00371 & 8.9 \\
\hline Band3 & 0.00450 & 0.00428 & 5.1 \\
\hline Band4 & 0.00305 & 0.00327 & -6.6 \\
\hline Band5 & 0.00105 & 0.00116 & -9.1 \\
\hline Band6 & 0.00172 & 0.00166 & 3.6 \\
\hline Band7 & 0.00444 & 0.00429 & 3.6 \\
\hline Band8 & 0.00452 & 0.00412 & -2.9 \\
\hline Band9 & 0.00306 & 0.00315 & -3.0 \\
\hline Band10 & 0.00108 & 0.00112 & 9.8 \\
\hline
\end{tabular}




\subsection{Dark level and dark noise}

The CAI-2 acquires dark calibration data by imaging the ocean around midnight. To avoid the influence of sunshine, the data is acquired during the time when the satellite is in the shade of the Earth. Examples of the obtained dark calibration data are shown in Figure 6. Although the fixed pattern of lines due to the variation of the dark signal of each pixel is clearly seen in the dark signal of Band5, these stripe patterns are corrected by performing dark subtraction.
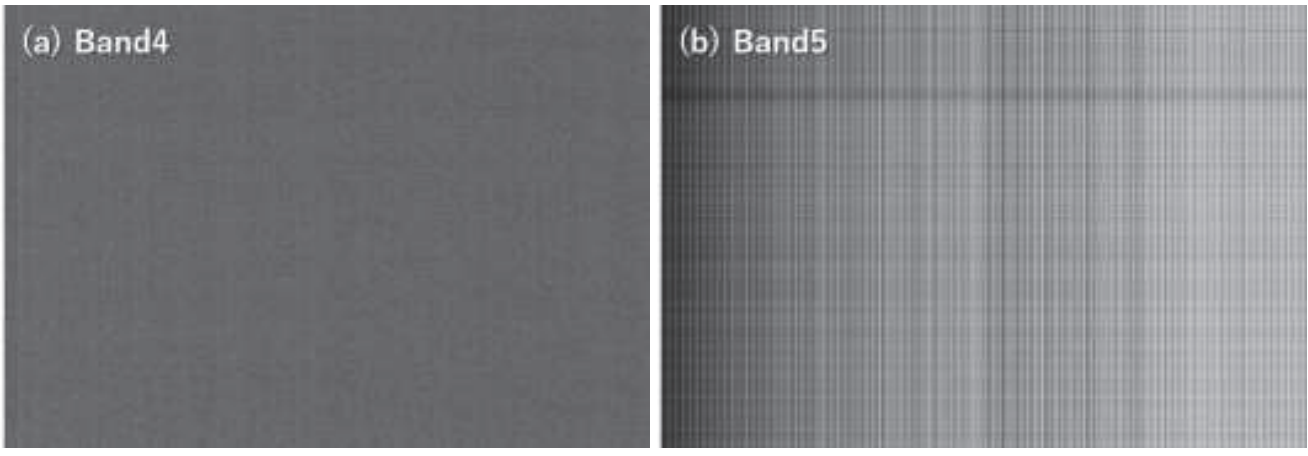

Figure 6. Examples of uncorrected raw dark signals taken above the mid-night ocean (December 2020). (a) Band4; (b) Band5. The stripe pattern of lines due to the variation of the dark signal of each pixel is clearly seen in the dark signal of Band5.

Bands 1 to 4 and 6 to 9 have 8 pre-scan pixels to monitor the variation of dark current. No pre-scan pixels exist in the InGaAs detectors for Bands 5 and 10, therefore the first six pixels of the 1024 pixels are masked to create a region of dark pixels. The dark current level is monitored by the output of these pre-scan pixels and masked pixels. The dark level of each band was evaluated by calculating the average value of all pixels for approximately 100 lines of raw data before pre-scan correction. The dark noise of each band was also evaluated by calculating the standard deviation $(1 \sigma)$ of approximately 100 lines of each pixel after subtracting the pre-scan or masked pixel data, by averaging the standard deviation for all pixels. Table 5 shows the comparison of the dark level and dark noise between the data obtained from the pre-flight test on the ground and the data obtained from the on-orbit dark calibration. There was no significant change in the dark level and dark noise from the ground test. It was confirmed that the performance at the ground test was maintained.

Table 5. The comparison of the dark level (digital number: DN) and the dark noise (standard deviation of digital number: $\mathrm{DN}$ rms) between the data obtained during the ground pre-flight test (PFT) and the on-orbit dark calibration at the beginning of the initial checkout phase (December 2018).

\begin{tabular}{|c|c|c|c|c|}
\hline \multirow{2}{*}{ Band } & \multicolumn{2}{|c|}{ Dark Level (DN) } & \multicolumn{2}{c|}{ Dark Noise (DN rms) } \\
\cline { 2 - 5 } & PFT & On-orbit & PFT & On-orbit \\
\hline Band1 & 50.2 & 52.5 & 0.95 & 0.82 \\
\hline Band2 & 50.2 & 49.2 & 0.92 & 0.80 \\
\hline Band3 & 52.3 & 49.0 & 0.86 & 0.76 \\
\hline Band4 & 51.7 & 52.9 & 0.89 & 0.77 \\
\hline Band5 & 76.8 & 85.7 & 2.78 & 2.65 \\
\hline Band6 & 52.2 & 55.0 & 0.95 & 0.84 \\
\hline Band7 & 49.3 & 50.6 & 0.95 & 0.84 \\
\hline Band8 & 48.0 & 49.9 & 0.93 & 0.86 \\
\hline Band9 & 48.3 & 49.2 & 0.96 & 0.85 \\
\hline Band10 & 71.8 & 81.9 & 2.79 & 2.64 \\
\hline
\end{tabular}




\section{NOMINAL OPERATION PHASE}

\subsection{Radiometric performance}

Following the initial calibration phase, the radiometric performance of the CAI-2 has been continuously evaluated. In this chapter, the evaluation results of the radiometric response trend data for two years after the initial calibration phase are presented. As described in Section 2.2, the comparison of on-orbit lunar observations with the ROLO model has a slightly large difference due to the inclusion of the effects of geometric uncertainties in the orbital position and attitude, as well as the uncertainties in the lunar reflectance model itself. The observed irradiance error of the Moon due to geometry such as lunar phase variation and orbital error affects all bands in the same ratio. In order to reduce the influence of such geometric factors, the observed irradiance in Bands 1, 2, 3, 5 and Bands 6, 7, 8, 10 were normalized by Bands 4 and 9, respectively, with a wavelength of $865 \mathrm{~nm}$, which is considered less affected by radiation and contamination. And the relative variation was calculated from the initial calibration phase in February 2019. The relative response change $\gamma$ from the initial calibration phase is derived by the following equation:

$$
\begin{gathered}
\alpha_{\text {Band }(x)}=H_{\text {Band }(x)} / H_{B a n d}^{\prime}(x) \\
\beta_{\text {Band }(x)}=\frac{\alpha_{\text {Band }(x)}}{\alpha_{B a n d}(4,9)} \\
\gamma_{\text {Band }(x)}=\frac{\left(\beta_{\text {Band }(x)}-\beta_{\text {Band }(x), \text { ref })}\right.}{\beta_{\text {Band }(x), \text { ref }}}
\end{gathered}
$$

where $\mathrm{x}$ is the index of each Band, $\alpha$ is the ratio of the observed lunar irradiance $H$ to the calculated irradiance $\mathrm{H}^{\prime}, \beta$ is the relative value of $\alpha$ for each band with respect to Band 4 for the forward bands and Band 9 for the backward bands, and $\gamma$ is the variation of $\beta$ at each calibration based on the initial calibration phase referred as $\beta_{\text {Band }(x), r e f}$. The calculated results of the relative response change $\gamma$ from the initial calibration phase are shown in Figure 7.
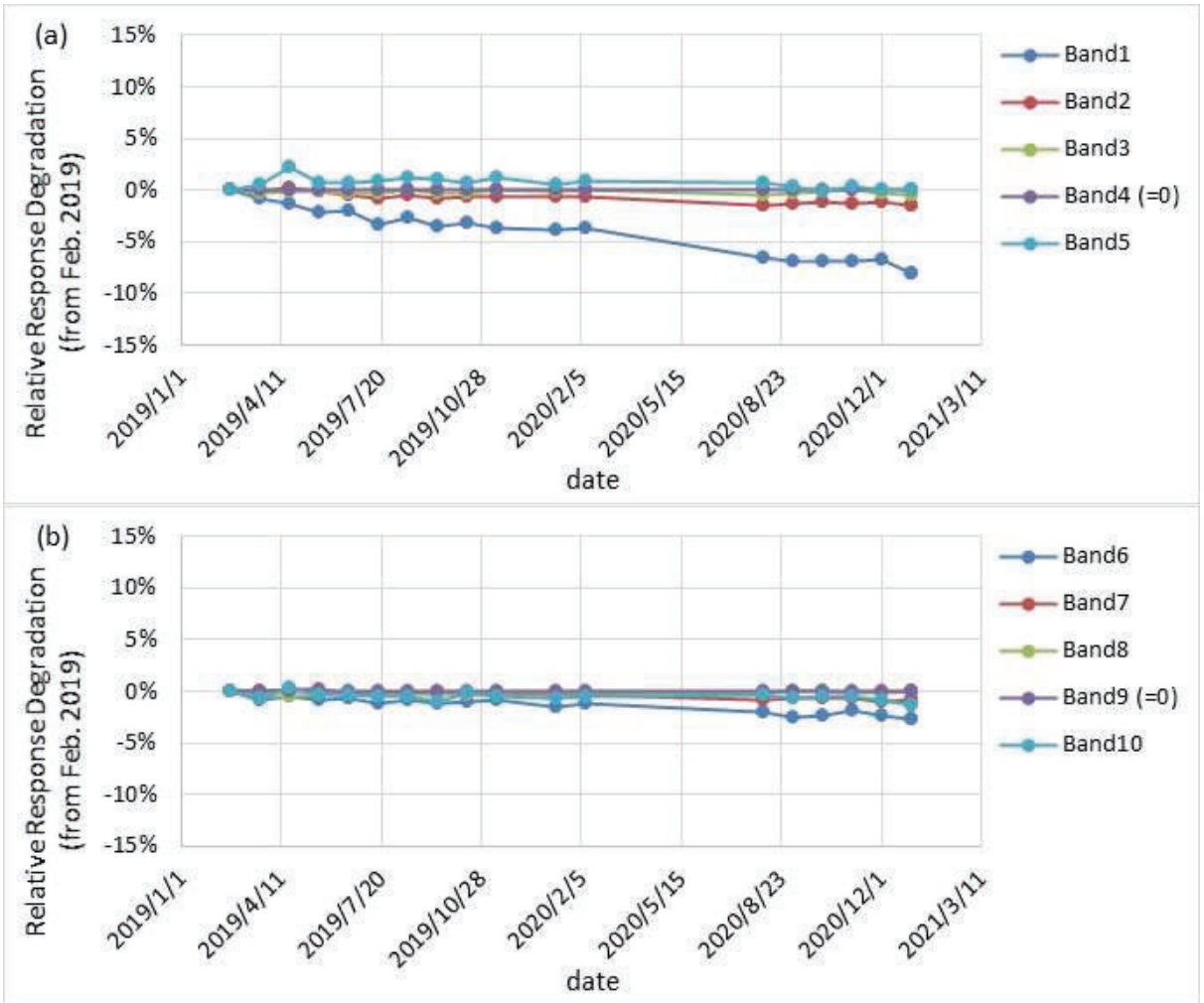

Figure 7. Results of the relative response change $\gamma$ from the initial calibration phase (February 2019). Forward Bands normalized by Band 4 (a) and Backward Bands normalized by Band 9 (b). The dark blue dots indicate Band 1 and Band 6. 
The radiometric response evaluation using the lunar calibration data and the ROLO model showed a response degradation in the UV Bands. Relative to Band 4 and Band $9(865 \mathrm{~nm})$, Band $1(340 \mathrm{~nm})$ and Band $6(380 \mathrm{~nm})$ showed a degradation of about $8 \%$ and $3 \%$ in two years, respectively. The degradation was found in shorter wavelengths of UV bands. It might be affected by contamination and UV radiation rather than the longer wavelength bands. In the other longer wavelength bands, the observed degradation was less than $1.5 \%$ relative to Band 4 or Band9. These results suggest that the radiometric performance of the CAI-2 is generally maintained for two years after initial calibration phase.

\subsection{Dark level and dark noise}

The evaluation of dark level and dark noise has been continuously carried out using the methods described in Section 2.3 . The trend graph is shown in Figure 8. Although there was some variation especially in Band 10, there was no significant increase in the dark level and the dark noise in all bands. Both the dark level and the dark noise are considered stable during the two years of evaluation from the initial calibration phase.
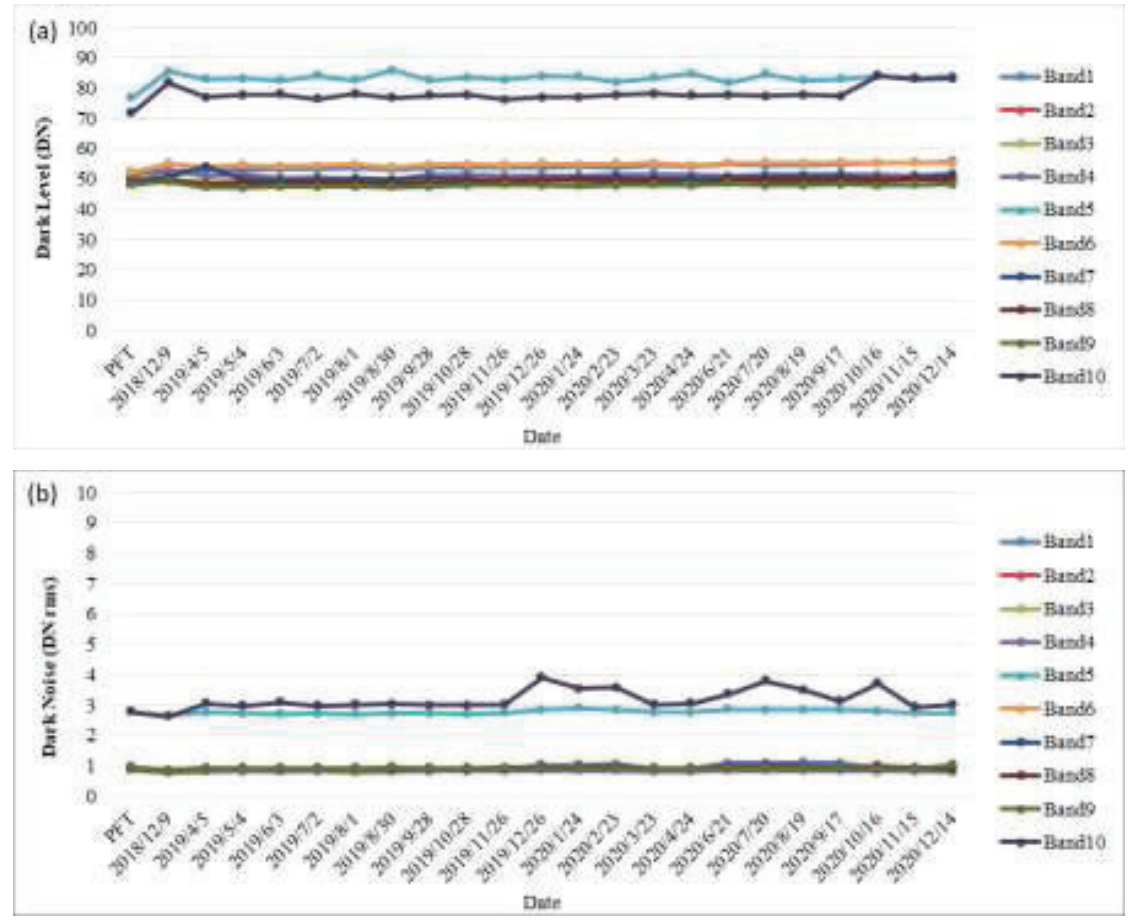

Figure 8. Trend graph of the dark level (a), and the dark noise (b). The dark violet dots indicate Band 10. Band 5 and Band 10 have slightly higher amounts of dark level and dark noise than the other bands due to the difference in detectors.

\section{CONCLUSION}

Since February 2019, the initial calibration phase was conducted to evaluate the geometric and radiometric properties of the CAI-2. Furthermore, following the initial calibration phase, the radiometric performance of the CAI-2 has been continuously evaluated. As for the evaluation of the two years since the initial calibration phase, although the dark level and the dark noise have shown some variation, the results have been stable with no significant increase. The radiometric response evaluation using the lunar calibration data and the ROLO model showed a response degradation in the UV Bands. Relative to Band 4 and Band $9(865 \mathrm{~nm})$, Band $1(340 \mathrm{~nm})$ and Band $6(380 \mathrm{~nm})$ showed a degradation of about $8 \%$ and $3 \%$ in two years, respectively. In the other longer wavelength bands, the observed degradation was less than $1.5 \%$ relative to Band 4 or Band9. From these results, it is considered that the radiometric performance of the CAI- 2 has been generally maintained in the two years since the initial calibration phase. 


\section{ACKNOWLEDGEMENTS}

GOSAT-2 is a joint project by Japan Aerospace Exploration Agency (JAXA), National Institute for Environmental Studies (NIES) and Ministry of the Environment (MOE). This work was conducted as a part of the GOSAT-2 operation funded by MOE.

\section{REFERENCES}

[1] GOSAT-2 Project. "About GOSAT-2", Slate, 27 February 2017, <http://www.gosat-2.nies.go.jp/about/mission> (8 February 2021).

[2] Nakajima, M., Suto, H., Yotsumoto, K., Shiomi, K., Hirabayashi, T., "Fourier transform spectrometer on GOSAT and GOSAT-2", Proc. of SPIE Vol. 105631056340 (2014).

[3] Suto, H., Kataoka, F., Kikuchi, N., Knuteson, R. O., Butz, A., Haun, M., Buijs, H., Shiomi, K., Imai, H., and Kuze, A.: Thermal and near-infrared sensor for carbon observation Fourier-transform spectrometer-2 (TANSOFTS-2) on the Greenhouse Gases Observing Satellite-2 (GOSAT-2) during its first year on orbit, Atmos. Meas. Tech. Discuss. [preprint], in review, 2020.

[4] Nakajima, M., Hashimoto, M., Sakai, M., Suto, H., Shiomi, K., Imai, H., Crawford, L., Davis, S. G., "Results of the commissioning phase of the mission instruments on GOSAT-2," Proc. SPIE 11151, 1115103 (2019).

[5] Kieffer, H. H. and Stone, T. C., "The spectral irradiance of the Moon", Astron. J., 129, pp. 2887-2901 (2005).

[6] Ono, A., Arai, K., Obata, K., Kuze, A., Kouyama, T., Sakuma, F., Tsuchida, S., Tonooka, H., Matsunaga, T., [Calibration of optical sensors learned from the basics], Rikoh Tosho, Tokyo, Japan, 81-88 (2020).

[7] C. Wehrli, "Spectral Solar Irradiance Data (WMO ITD 149; Geneva: WMO)" (1986).

[8] Stone, T.C. and Kieffer, H.H. "Assessment of Uncertainty in ROLO Lunar Irradiance for On-orbit Calibration", Proc.SPIE2004, 5542, 300-310 (2004). 